\title{
Cámaras trampa como método de muestreo para aves del Chaco Seco paraguayo: una comparación con los métodos auditivos y visuales
}

\author{
Griselda Inés Zárate-Betzel ${ }^{1 *}$, Andrea Weiler Gustafson², Karina Beatriz Núñez Goralewski², \\ Alberto Esquivel Mattos ${ }^{3}$, Stella Mary Amarilla Rodríguez ${ }^{4} \&$ Juan Manuel Pech-Canché $^{5}$ \\ 1. Universidad Nacional de Asunción, Facultad de Ciencias Agrarias, Maestría en Manejo de Recursos Naturales y \\ Gestión Ambiental del Territorio; grisel.zb@gmail.com \\ 2. Universidad Nacional de Asunción, Facultad de Ciencias Exactas y Naturales, Departamento de Biología; \\ andreaweiler1@gmail.com, ranitapy@gmail.com \\ 3. Wildlife Tours Paraguay, Palma Loma, Luque; alberto.esquivel@wildlife.com.py \\ 4. Universidad Nacional de Asunción, Facultad de Ciencias Agrarias, Dirección de Postgrado; \\ stella.amarilla@agr.una.py \\ 5. Universidad Veracruzana, Facultad de Ciencias Biológicas y Agropecuarias, Laboratorio de Vertebrados Terrestres; \\ jmpech@gmail.com \\ * Correspondencia
}

Recibido 10-X-2018. Corregido 15-VII-2019. Aceptado 09-VIII-2019.

\begin{abstract}
Camera traps as a sampling method for Paraguayan Dry Chaco birds: a comparison with auditory and visual methods. Camera trap as a sampling method for Paraguayan Dry Chaco birds: a comparison with auditory and visual methods. Camera trap techniques are widely used for the study of mammals, but also enable investigation of other groups, such as birds. In order to evaluate the usefulness of camera trapping for the study of avifauna, we analyzed the records obtained from 12 camera traps installed between July 2015 and July 2016, near artificial water sources in Estancia Montania, Boquerón Department, Paraguay. Four of these sites were associated with xerophytic forests and eight with exotic pastures. The richness, composition and capture rate recorded in both ecosystems were determined. We compared the richness and composition of birds detected by camera traps with those obtained through auditory and visual methods. Through camera trapping, 74 species of birds were recorded, belonging to 30 families and 16 orders. The xerophytic forest and the exotic pasture have a $52.7 \%$ similarity in species composition. With regard to capture rates, the most frequently recorded species were: Leptotila verreauxi (16.14\%), Ortalis canicollis (14.28\%) and Furnarius cristatus (11.39\%). The camera traps were effective in detecting $33 \%$ of the species identified in the area based on direct and auditory observation sampling techniques, and added two species not detected by direct methods. For this reason, camera trapping is appropriate to complement methods based on direct observation, particularly for monitoring hunted species of the Chaco ecosystem belonging to Tinamiform and Columbiform species. Due to the non-invasive nature of the technique, it constitutes an efficient method for addressing ethological studies of terrestrial species.
\end{abstract}

Key words: camera trapping; species richness; terrestrial birds; diversity; Tinamiform.

Zárate-Betzel, G. I., Weiler Gustafson, A., Núñez Goralewski, K. B., Esquivel Mattos, A., Amarilla Rodríguez, S. M., \& Pech-Canché, J. M. (2019). Cámaras trampa como método de muestreo para aves del Chaco Seco paraguayo: una comparación con los métodos auditivos y visuales. Revista de Biología Tropical, 67(4), 1089-1102.

El Gran Chaco Americano abarca parte del territorio de los países de Argentina, Bolivia, Paraguay y Brasil. Considerando las comunidades vegetales y el régimen de precipitación anual del área se divide en dos ecorregiones: el Chaco Húmedo y el Chaco Seco (Dinerstein et al., 1995). Dichas ecorregiones han sufrido aceleradas modificaciones en las últimas 
décadas, causadas principalmente por el desarrollo de actividades agropecuarias, alcanzado una tasa de deforestación de 1376 ha por día en el año 2013 (Caballero, Palacios, Arévalos, Rodas, \& Yanosky, 2014). Esto ha ocasionado la transformación de bosques xerofíticos en pasturas y la construcción de aguadas artificiales, consistentes en reservorios de agua que garantizan su disponibilidad durante el año.

Una técnica muy utilizada en la actualidad para el estudio de vertebrados terrestres de la región es el fototrampeo, debido a su practicidad, calidad y la cantidad de información que provee (Belda, Arques, Martínez, Peiró, \& Seva, 2009). Posee ventajas con respecto a métodos de muestreo sistemático como los transectos, la captura y recaptura, y los puntos de conteo debido a que, a pesar de que los mismos se encuentran bien desarrollados, pueden ser difíciles de replicar y estandarizar a causa de diferencias en el esfuerzo de muestreo, sesgos en la observación y entrenamientos inadecuados. En contrapartida, mediante el fototrampeo la influencia humana y el error se reducen a la instalación y el mantenimiento de las cámaras trampa y la identificación de las fotografías obtenidas (Ahumada, Hurtado, \& Lizcano, 2013). Así también, el fototrampeo es considerado ideal para la detección de especies crípticas, raras y nocturnas, además de especies amenazadas, cuya captura o colecta está restringida o prohibida (Wemmer, Kunz, LundieJenkins, \& McShea, 1996; Dinata, Nugroho, Haidir, \& Linkie, 2008).

Las cámaras trampa son utilizadas principalmente en estudios diseñados para la toma de datos referentes a mamíferos medianos y grandes (Karanth \& Nichols, 1998; Karanth, Nichols, Kumar, Link, \& Hines, 2004; Mccallum, 2013). En estos muestreos se obtienen además registros de otros grupos taxonómicos, principalmente de aves terrestres, los cuales son descartados por no proveer información vinculada a los objetivos de las investigaciones. Estos datos podrían ser utilizados para ampliar el conocimiento sobre la ecología e historia natural de las especies, aprovechando el esfuerzo y los recursos materiales invertidos.
Los muestreos de aves se realizan frecuentemente a través de métodos como los puntos de conteo, transectos, y listas Mackinnon, en los cuales se registran las especies vistas y oídas (Mackinnon \& Phillipps, 1993; González-García, 2012). Por otra parte, los estudios mediante cámaras trampa han sido escasos, pero han aportado información sobre la riqueza, la distribución, los patrones de actividad, la abundancia relativa y ocupación de aves (Dinata et al., 2008; O'Brien \& Kinnaird, 2008; Belda et al., 2009; Arnulphi, Ortiz, \& Borghi, 2013; Mosquera-Muñoz, Corredor, Cardona, \& Armbrecht, 2015). Algunos autores han utilizado el fototrampeo para estudiar la depredación de nidos (Bayne \& Hobson, 1997; Buler \& Hamilton, 2000), y los mecanismos de defensa parental de los mismos (Bradley \& Marzluff, 2003; Pietz \& Granfors, 2005). Así también, este método ha sido utilizado para la identificación de frugívoros dispersores de semillas, incluidas las aves (Miura, Yasuda, \& Ratnam, 1997; Rojas-Robles, Stiles, \& MuñozSaba, 2012). A través del uso de cámaras trampa se han realizado registros de aves amenazadas como Aquila chrysaetos (Accipitridae) (Guerrero-Cárdenas, Galina-Tessaro, ÁlvarezCárdenas, \& Mesa-Zavala, 2012) y Carpococcyx viridis (Cuculidae) (Zetra, Rafiastanto, Rombang, \& Trainor, 2002).

Las aves pueden ser clasificadas según el lugar en el cual buscan su alimento en los siguientes gremios: aéreas, arborícolas, acuáticas y terrestres (De La Peña \& Krüger, 1987). Las cámaras trampas han sido utilizadas mayormente para el estudio de aves terrestres y acuáticas, de tamaño grande, especialmente pertenecientes a los órdenes Galliformes, Gruiformes y Tinamiformes (Li, Mcshea, Wang, Shao, \& Shi, 2010; Armenteros, Prieto, Lomillos, Alonso, \& Gaudioso, 2015; Mena, Zúñiga Hartley, Villacorta Pezo, \& Salazar Zorrilla, 2016; Colyn, Campbell, \& Smit-Robinson, 2017).

El objetivo general de la presente investigación fue valorar la utilidad de las cámaras trampa en el estudio de la avifauna. Para ello, se identificó la riqueza y composición de 
especies de aves presentes en bosques xerofíticos y pasturas exóticas, el grado de cambio en la composición de especies entre comunidades y la tasa de captura registrada mediante fototrampeo en los boques xerofíticos y en las pasturas exóticas. Así también, se comparó la riqueza y composición de especies identificadas a través de las cámaras trampa con la registrada mediante listas Mackinnon.

\section{MATERIALES Y MÉTODOS}

Área de estudio: La investigación fue realizada en el establecimiento ganadero Estancia Montania (215'29” S \& 6004'56" W) departamento de Boquerón, Región Occidental del Paraguay. Dicho departamento se caracteriza por carecer de accidentes orográficos importantes, y una elevación de aproximadamente $100 \mathrm{~m}$ sobre el nivel del mar.

La Estancia Montania tiene una superficie de 37000 ha, de las cuales 10410 ha corresponden a bosques xerofíticos con especies vegetales dominantes del estrato superior como Schinopsis lorentzii (Anacardiaceae), Aspidosperma quebracho-blanco (Apocynaceae), Ceiba chodatii (Malvaceae), y un pequeño palmar de Copernicia alba (Arecaceae). Las 26590 ha restantes han sido convertidas a pasturas exóticas de Panicum maximum (Poaceae), manteniéndose cortinas de vegetación arbórea de 80 a $100 \mathrm{~m}$ de ancho, que sirven de rompevientos. El establecimiento posee un total de 28 aguadas artificiales, distribuidas en zonas próximas a los bosques xerofíticos y pasturas exóticas.

Fototrampeo: Los datos utilizados fueron obtenidos en el marco del proyecto de investigación "14 INV 187- Determinación del valor de paisajes ganaderos en la conservación de la biodiversidad en el Chaco Seco paraguayo" el cual fue financiado por el Consejo Nacional de Ciencia y Tecnología del Paraguay. Los registros se realizaron de julio de 2015 a julio de 2016, mediante 12 cámaras trampa automáticas ubicadas a pocos metros de aguadas artificiales permanentes, cuatro asociadas a bosque xerofítico, y ocho a pasturas exóticas. Las mismas fueron colocadas aproximadamente a 50 $\mathrm{cm}$ del suelo, con una distancia mínima de 1.5 $\mathrm{km}$ entre cada estación de muestreo. Tres cámaras trampa correspondieron a la marca Bushnell y nueve a la marca Velleman, todas contaban con sensores infrarrojos. Fueron programadas para funcionar las $24 \mathrm{~h}$ del día, y tomar tres fotografías y un video de diez segundos por cada detección, con intervalos de $30 \mathrm{~s}$. Fueron revisadas aproximadamente cada 30 días para el cambio de baterías y tarjetas de memoria.

Análisis de datos: Se cuantificaron los días en los cuales las cámaras trampa permanecieron activas, y el número de capturas (fotografías y videos) de aves de cada sitio, con la finalidad de determinar el esfuerzo de muestreo. Para el análisis de los datos se seleccionaron las capturas efectivas, es decir, en las cuales fue posible identificar a la especie registrada. En el ordenamiento taxonómico se siguió a Clements et al. (2016).

Se realizó una curva de acumulación utilizando los estimadores no paramétricos Jacknife 1 y Jacknife 2, mediante el programa Estimates 9.1.0 (Colwell, 2013). El grado de cambio o reemplazo en la composición de especies entre comunidades del paisaje (diversidad beta) fue estimado mediante el coeficiente de similitud de Jaccard (Moreno, 2001). La tasa de captura se calculó a partir del número de días en los cuales se registró la presencia de la especie sobre el total de días en los cuales la cámara trampa permaneció activa por 100 (Moreira et al., 2007).

Se comparó el número de especies, las familias y los órdenes de aves registrados mediante cámaras trampa con los resultados obtenidos por Weiler, Esquivel, Peris, Silla y Salinas (2018) quienes realizaron listas Mackinnon (Mackinnon \& Phillipps, 1993). Dicho método consistió en registrar las especies identificadas visual o auditivamente en listas de diez especies. Los muestreos fueron bimensuales, y abarcaron la temporada seca y lluviosa durante el 2016 y 2017. Cada muestreo tuvo una duración de siete días. 


\section{RESULTADOS}

Riqueza y composición de especies: Con un esfuerzo de muestreo total de 2317 días trampa (57\% del esfuerzo en pasturas y $43 \%$ en bosques xerofíticos), se obtuvo un total de 159144 capturas (fotografías y videos), de los cuales 20597 correspondieron a capturas efectivas. Se registraron 74 especies de aves, pertenecientes a 30 familias y 16 órdenes (Apéndice 1).

De las 18 especies de aves endémicas del Gran Chaco Americano que ocurren en Paraguay, nueve fueron registradas mediante el fototrampeo en este estudio. Guyra Paraguay (2005) indica que 65 de estas especies son residentes permanentes del país, tres nidificantes migrantes del sur, tres migradores australes, dos visitantes invernales y un migrador neártico (Apéndice). Todas las especies corresponden a la categoría "Preocupación Menor" de la Unión Internacional para la Conservación de la Naturaleza (IUCN, 2017).

Las curvas de acumulación de especies, tanto de bosques xerofíticos como de pasturas exóticas, muestran que un aumento en el número de unidades de muestreo (días trampa) incrementarán la cantidad de especies observadas (Fig. 1 y Fig. 2) pudiendo registrarse de 15 a 24 especies más en el bosque xerofítico. Con respecto a las pasturas exóticas, los estimadores de riqueza indican que pueden registrarse de 17 a 31 especies adicionales.

Composición de especies entre comunidades: El coeficiente de similitud de Jaccard indica que los bosques xerofíticos y pasturas exóticas poseen un $52.7 \%$ de similitud en cuanto a la composición de las especies registradas mediante fototrampeo. En los bosques fueron registradas 54 especies de aves, mientras que en las pasturas la riqueza fue de 59. Estos sitios comparten un total de 39 especies, entre las cuales se encuentran aves de hábitos terrestres como Nothoprocta cinerascens, Nothura boraquira y Crypturellus tataupa (Tinamidae), Ortalis canicollis (Cracidae), Chunga burmeisteri (Cariamidae) y Aramides ypecaha (Rallidae). Fueron registradas 15 especies únicamente en los bosques, como

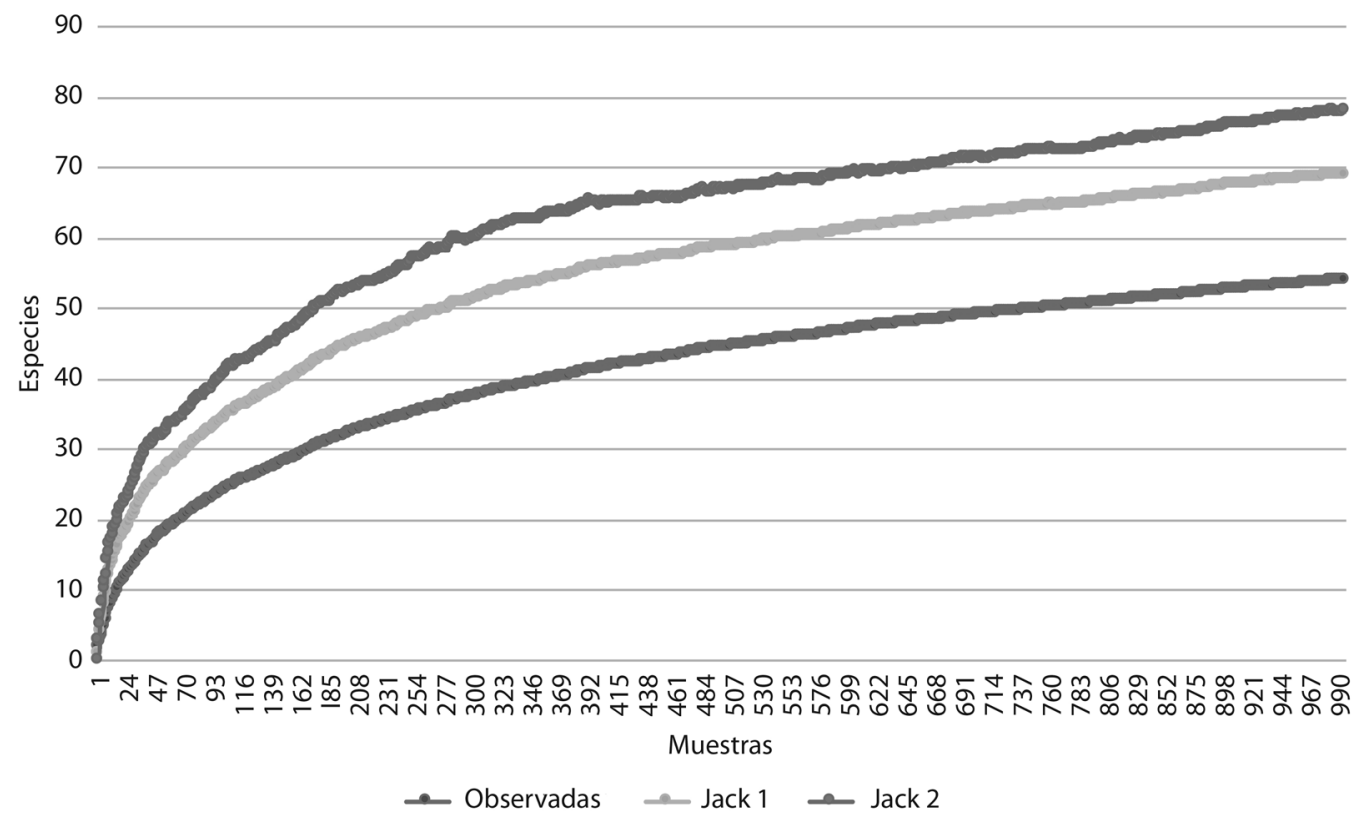

Fig. 1. Curva de acumulación de especies en bosques xerofíticos

Fig. 1. Species accumulation curves in xerophytic forest. 


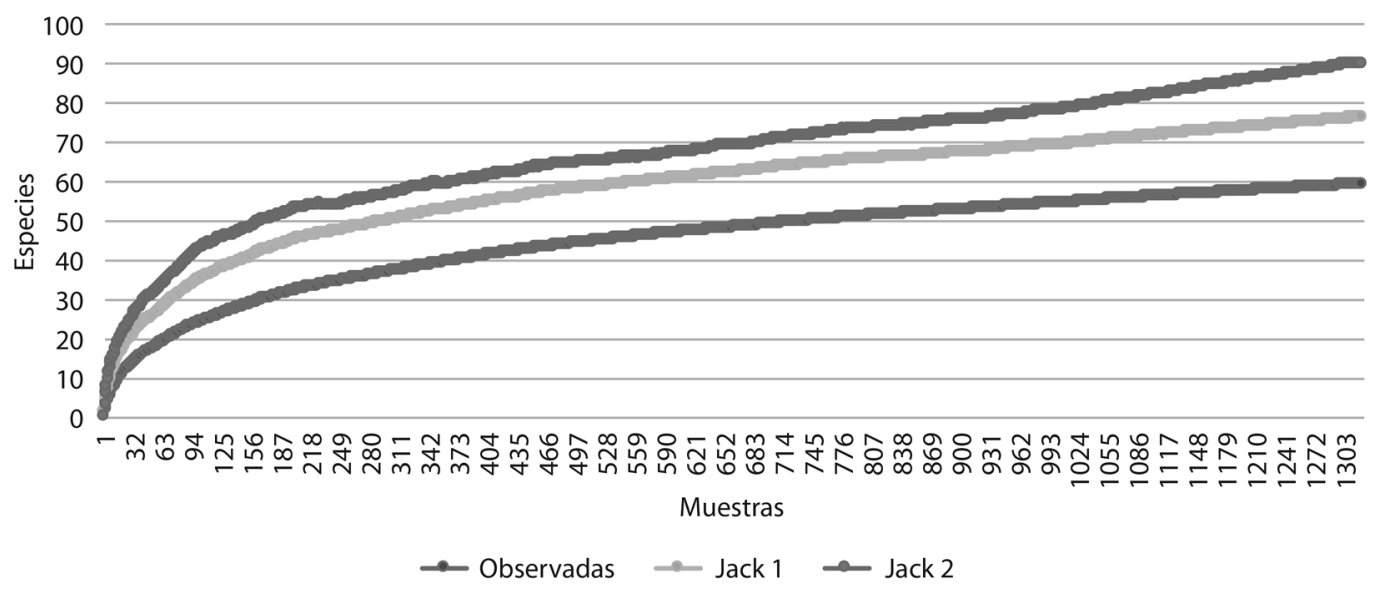

Fig. 2. Curva de acumulación de especies en pasturas exóticas.

Fig. 2. Species accumulation curves in exotic pastures.

Aramides cajanea (Rallidae); mientras que 20 especies fueron registradas exclusivamente en las pasturas, entre las cuales se encuentran Nothura chacoensis (Tinamidae), y Drymornis bridgesii (Dendrocolaptidae) (Apéndice).

Tasa de captura: En los bosques xerofíticos, las especies con mayor tasa de captura fueron: Ortalis canicollis (19.59\%), Leptotila verreauxi (16.29\%), Furnarius cristatus (11.82 \%), Aramides ypecaha (5.965 \%), Columbina picui (5.75\%), Paroaria coronata (4.68 $\%)$ y Coryphistera alaudina (4.36\%). Las menores tasas de captura, constituidas por un único registro, corresponden a Ardea alba, Egretta thula, Syrigma sibilatrix, Geranospiza caerulescens, Jacana jacana, Tringa solitaria, Patagioenas cayennensis, Bubo virginianus, Colaptes melanochloros, Xiphocolaptes major, Fluvicola albiventer, Pyrocephalus rubinus, Cyclarhis gujanensis, Sicalis flaveola y Cyanocompsa brissonii (Fig. 3).

En las pasturas exóticas, las mayores tasas de captura corresponden a Leptotila verreauxi (17.26 \%), Furnarius cristatus (11.95 \%), Ortalis canicollis (11.48 \%), Aramides ypecaha (11.48 \%), Columbina picui (8.43\%), Coryphistera alaudina (6.17\%) y Turdus amaurochalinus (4.29\%), mientras que las especies detectadas en una única ocasión fueron
Eudromia formosa, Crypturellus tataupa, Butorides striatus, Ardea alba, Nycticorax nycticorax, Cairina moschata, Rupornis magnirostris, Geranospiza caerulescens, Crotophaga ani, Crotophaga major, Lepidocolaptes angustirostris, Elaenia spectabilis, Myiodynastes maculatus, Knipolegus striaticeps, Cyanocorax chrysops, Saltator multicolor y Coryphospingus cucullatus (Fig. 3).

Comparación de los resultados del fototrampeo y listas Mackinnon: Weiler et al. (2018) registraron mediante listas Mackinnon 216 especies de aves, pertenecientes a 23 órdenes y 52 familias. Las cámaras trampa resultaron efectivas para el registro de 74 especies, de las cuales dos, Eudromia formosa (Tinamidae) y Patagioenas cayennensis (Columbidae), no fueron inventariadas a través de listas Mackinnon. Combinando ambos resultados, la riqueza del área de estudio asciende a 218 especies de aves.

Las cámaras trampa registraron un mayor número de especies de los órdenes Tinamiformes y Columbiformes que las listas Mackinnon. Esto indica que el fototrampeo constituye un método apropiado para la detección de los taxones referidos. Las especies de los demás órdenes fueron registradas mayormente a través de listas Mackinnon, por lo cual este 


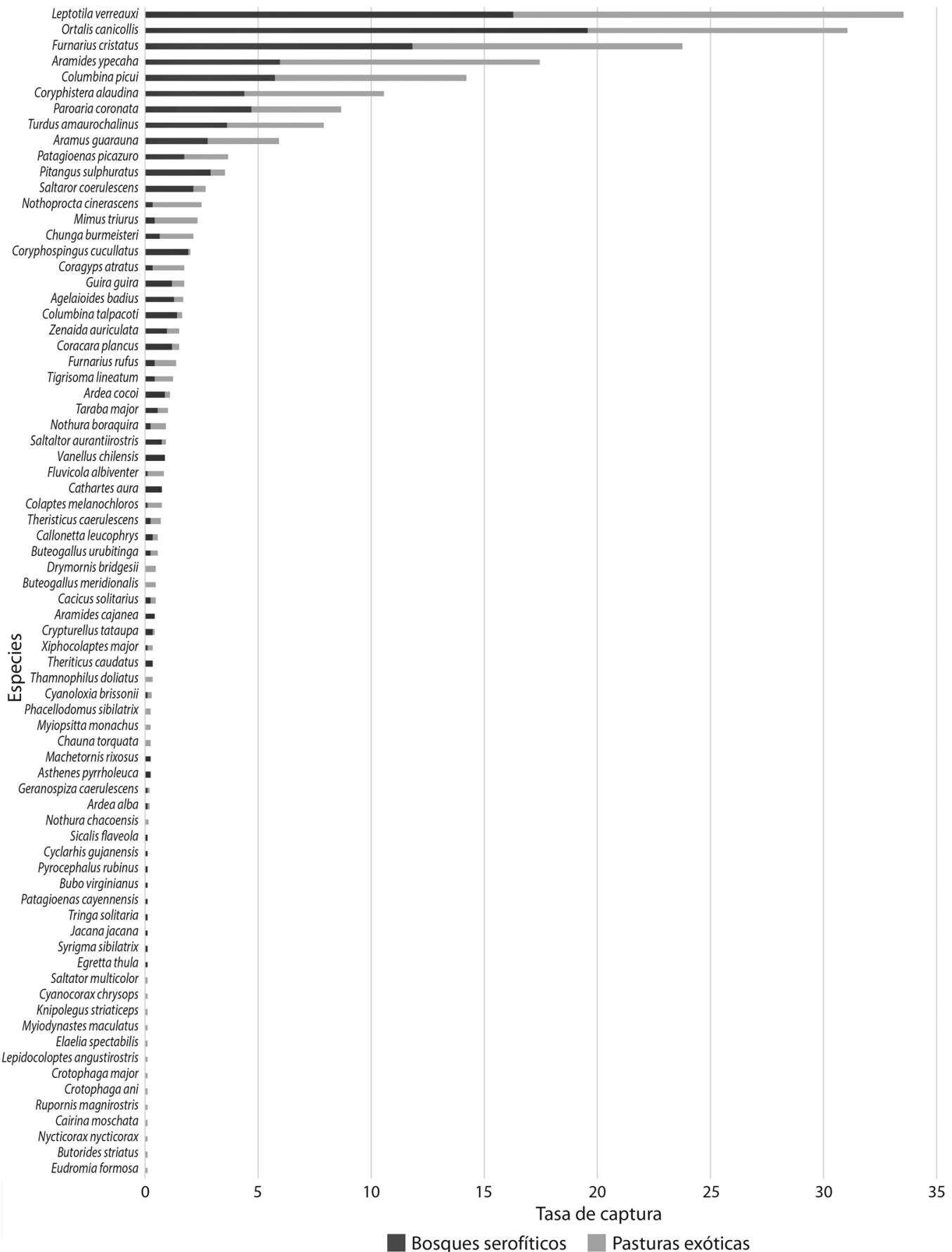

Fig. 3. Tasa de captura en bosques xerofíticos y pasturas exóticas.

Fig. 3. Capture rate in xerophytic forests and exotic pastures. 


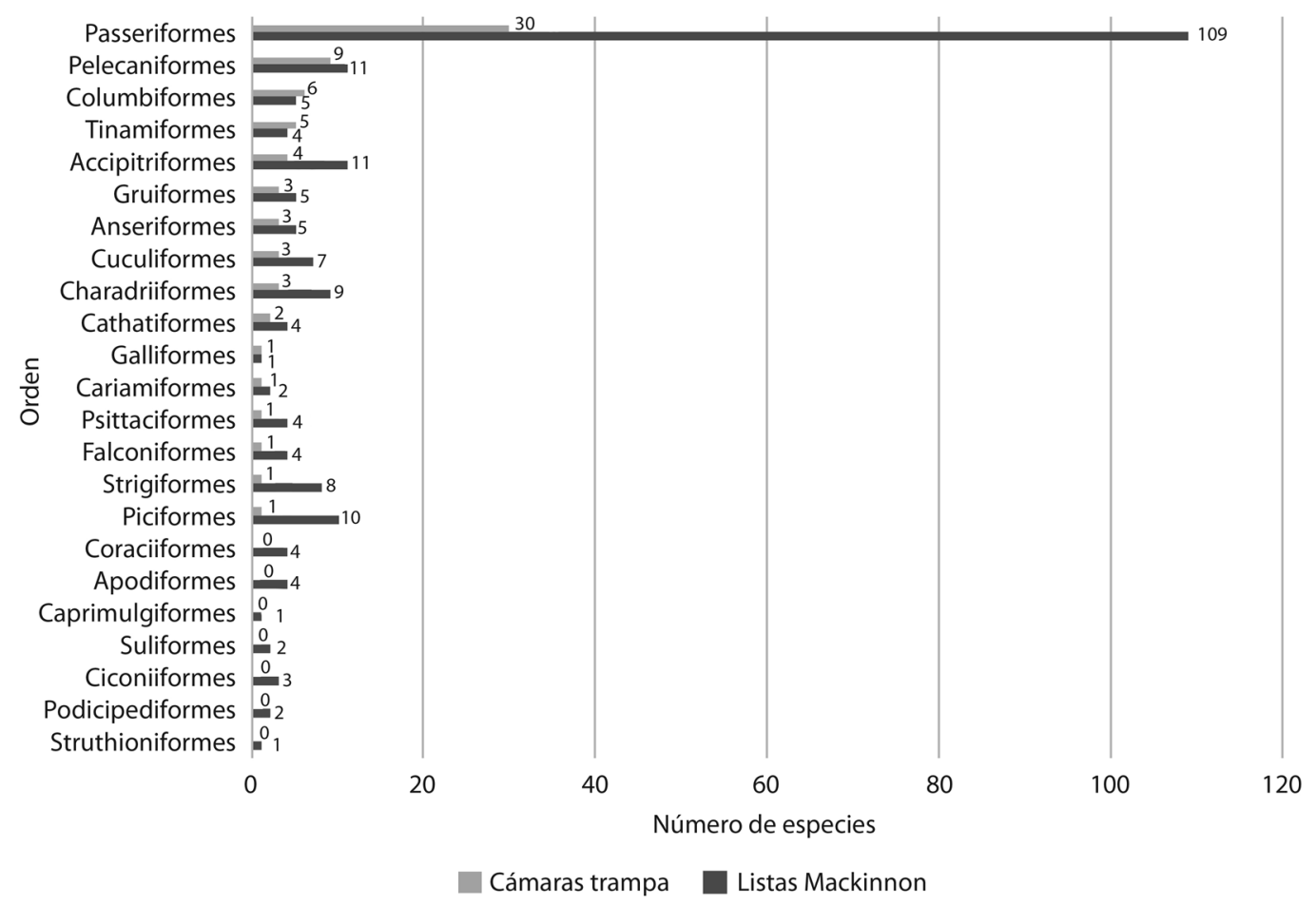

Fig. 4. Número de especies de cada orden registradas mediante listas Mackinnon y cámaras trampa.

Fig. 4. Number of species of each order recorded through Mackinnon lists and trap cameras.

método fue más efectivo para determinar la diversidad del área de estudio (Fig. 4).

\section{DISCUSIÓN}

La curva de acumulación y los estimadores indican que es posible aumentar el número de especies registradas. Los estimadores de riqueza utilizados se basan principalmente en el número de especies que sólo fueron registradas en una o dos muestras (uniques y duplicates). Esto se basa en el supuesto de que en los ecosistemas no existen individuos solos, sino poblaciones, por lo tanto un alto número de uniques y duplicates en el muestreo indica que no se han realizado suficientes repeticiones (Villareal et al., 2006). En el muestreo realizado, un total de 22 especies fueron registradas en sólo una unidad de muestreo, mientras que cinco especies fueron registradas en sólo dos unidades muestrales. Dichos resultados sugieren que el $36 \%$ de las especies observadas pueden ser consideradas raras (por su baja detección o su escasa abundancia relativa).

Con respecto a la riqueza de especies entre hábitats, el número de especies en pasturas exóticas fue mayor al del bosque xerofítico. Estos resultados difieren de los obtenidos por Mastrangelo y Gavin (2012) y Coria, Coria y Kunst (2016) en el Chaco Seco argentino, quienes registraron una menor riqueza de especies en pasturas. Así también, Tews et al. (2004) señalan que hábitats estructuralmente complejos, como los bosques, albergan una mayor diversidad de especies debido a que proveen más nichos y diversidad de maneras de explotar los recursos ambientales.

No obstante, las pasturas del área de estudio constituyen un hábitat heterogéneo, debido a que se encuentran rodeadas por cortinas de vegetación arbórea. Estos fragmentos de vegetación nativa proveen recursos que son utilizados por algunas especies (Gascon et al., 1999; Ranganathan, Daniels, Chandran, Ehrlich, \& 
Daily, 2008; Herrera, 2011). Por lo tanto, la matriz variada en la cual se encuentran inmersas las pasturas exóticas pudo ser determinante para que albergue una riqueza de especies similar a la del bosque xerofítico.

Las mayores tasas de captura correspondieron a especies terrestres o caminadoras, como L. verreauxi, O. canicollis, F. cristatus y A. ypecaha. Con tasas de captura intermedias y bajas, fueron registradas especies acuáticas como Trigrisoma lineatum, Ardea cocoi y Theristicus caerulescens, Jacana jacana y Nycticorax nycticorax. Durante la temporada de lluvia, se registró la presencia de Callonetta leucophrys en las estaciones de muestreo inundadas. Colyn et al. (2017) mencionan la utilidad del fototrampeo para detectar y monitorear aves vinculadas a humedales.

Especies descritas como arborícolas y aéreas por Short (1975) como R. magnirostris, L. angustirostris, P. rubinus y C. gujanensis obtuvieron una baja tasa de captura. Esta no puede ser relacionada con la abundancia de las especies, debido a que la posición de las cámaras trampa no era apropiada para inventariar los mencionados gremios.

El $33 \%$ de la avifauna registrada a través de métodos de observación directa y auditiva fue detectada mediante las cámaras trampa, resultando éstas más efectivas para determinar la riqueza de especies de los órdenes Tinamiformes y Columbiformes. En el presente estudio se identificaron cinco especies de perdices (Tinamidae) a través del fototrampeo, mientras que mediante listas Mackinnon se registraron cuatro especies. La especie registrada únicamente mediante fototrampeo, en una sola ocasión, fue E. formosa, perdíz semigregaria endémica del Chaco (Short, 1975). Sus poblaciones son consideradas escasas y espaciadas (Contreras et al., 2014) y los resultados obtenidos sugieren una baja abundancia relativa en el área de estudio.

El orden Tinamiformes agrupa a perdices de plumaje compacto y mimético, lo cual puede constituir una dificultad para lograr el avistamiento directo de las especies (Narosky \& Yzurieta, 2006). Mena et al. (2016) obtuvieron resultados similares al realizar un muestreo con cámaras trampa en la cuenca alta del río La Novia (Perú), registrando una alta diversidad de perdices. En dicha investigación aportaron un primer registro para la zona, la especie Tinamus guttatus (Tinamidae), la cual no había sido registrada mediante listas Mackinnon y redes de niebla por Angulo, Novoa y Lazo (2016). Por su parte, Mosquera-Muñoz et al. (2015) ampliaron el área de distribución de Odontophorus hyperythrus (Odontophoridae), perdiz considerada casi amenazada, mediante registros obtenidos por cámaras trampa.

El orden Columbiformes agrupa a palomas que, si bien realizan vuelos sostenidos, frecuentan el suelo con la finalidad de alimentarse (Narosky \& Yzurieta, 2006). Las cámaras trampa capturaron imágenes de seis especies pertenecientes a dicho orden, una especie más que las registradas mediante listas Mackinnon. Las tasas de captura del fototrampeo han sido altas para especies como L. verreauxi, C. picui y Patagioenas picazuro. La especie registrada únicamente mediante fototrampeo fue $P$. cayennensis. Narosky y Yzurieta (2006) indican que dicha especie vuela y posa alto, por lo cual el registro obtenido puede ser considerado un evento fortuito.

El orden Galliformes, registrado por ambos métodos de muestreo, se encuentra representado en el área de estudio únicamente por la perdiz $O$. canicollis. La misma se caracteriza por ser terrestre y arborícola (Contreras et al., 2014) y ha obtenido una alta tasa de captura mediante el fototrampeo. Li et al. (2010) resaltan la utilidad del fototrampeo para el estudio de aves pertenecientes al orden Galliformes, con base en los resultados obtenidos en una investigación realizada en dos Reservas Naturales en la Provincia Sichuan, China. Los datos proveídos por las cámaras trampas permitieron el análisis de la ocupación y patrones de actividad de especies elusivas a la presencia humana.

Cabe destacar que los órdenes Tinamiformes, Columbiformes y Galliformes agrupan especies de aves consideradas predilectas para la caza en el Chaco argentino (Mosa \& Goytia, 2004), lo cual sugiere que el fototrampeo es 
un método de muestreo con potencial para el estudio de aves de interés socio-económico. Así mismo Mesa-Zavala, Alvarez-Cardenas, Galina-Tessaro, Troyo-Dieguez y GuerreroCardenas (2012), recalcan la utilidad del fototrampeo como método de muestreo para el estudio de especies cinegéticas, con base en los resultados obtenidos en una investigación realizada en Baja California Sur (México). Por otra parte, especies terrestres de los órdenes Struthioniformes y Caprimulgiformes no fueron registradas mediante el fototrampeo, mientras que a través de las listas Mackinnon se registró una especie de cada orden.

Las listas Mackinnon, los puntos de conteo y los transectos poseen la ventaja de permitir al investigador registrar aves situadas en diferentes estratos del dosel, a diferencia de las cámaras trampa, las cuales poseen un área de detección limitada. Además, debe considerarse que al permanecer estáticas durante largos periodos de muestreo aumenta la posibilidad de que estas últimas registren los mismos individuos, especialmente a las especies territoriales. En contrapartida, el monitoreo de la fauna por medio del fototrampeo posee ventajas sobre métodos que implican la observación directa de los ejemplares, destacándose la baja perturbación humana (O'Connell, Nichols, \& Karanth, 2010). Por este motivo, resulta apropiado para el registro de especies elusivas y el desarrollo de estudios etológicos. Las cámaras trampa constituyen una herramienta de utilidad para complementar métodos basados en la observación directa y auditiva de aves, resultando particularmente adecuadas para el abordaje de investigaciones enfocadas en especies terrestres como las pertenecientes al orden Tinamiformes.

En vista de que el uso de las cámaras trampa se ha extendido, principalmente con la finalidad de estudiar mamíferos, se recomienda utilizar los registros obtenidos por las mismas para aumentar los conocimientos sobre la distribución, diversidad, ocupación, abundancia y estructura de poblaciones de aves. Así también, se sugiere la realización de investigaciones enfocadas en el comportamiento de las especies, ya que constituye un método idóneo por no ocasionar perturbaciones a la vida silvestre durante su uso.

Declaración de ética: los autores declaran que todos están de acuerdo con esta publicación y que han hecho aportes que justifican su autoría; que no hay conflicto de interés de ningún tipo; y que han cumplido con todos los requisitos y procedimientos éticos y legales pertinentes. Todas las fuentes de financiamiento se detallan plena y claramente en la sección de agradecimientos. El respectivo documento legal firmado se encuentra en los archivos de la revista.

\section{AGRADECIMIENTOS}

Al Consejo Nacional de Ciencia y Tecnología por la beca otorgada para la realización de la Maestría y la financiación del Proyecto 14-INV-187: Determinación del valor de paisajes ganaderos en la conservación de la biodiversidad del Chaco Seco paraguayo. A los propietarios y al personal de la Estancia Montania por el apoyo a la investigación científica. A la Colección Zoológica de la Facultad de Ciencias Exactas y Naturales de la Universidad Nacional de Asunción. A Robert Owen por la revisión del abstract. A los revisores anónimos por sus valiosos comentarios y recomendaciones.

\section{RESUMEN}

Las cámaras trampa constituyen una herramienta de gran utilidad para el estudio de vertebrados terrestres. Con el objetivo de evaluar la utilidad del fototrampeo para el estudio de la avifauna, se analizaron los registros obtenidos, entre julio 2015 y julio 2016, por cámaras trampa instaladas en 12 puntos con aguadas artificiales de la Estancia Montania, departamento de Boquerón, Paraguay. Se determinó la riqueza, composición y tasa de captura registrada en bosques xerofíticos y pasturas exóticas. Se comparó la riqueza y composición de aves detectada mediante cámaras trampa con la obtenida a través de listas Mackinnon. Mediante fototrampeo, fueron registradas 74 especies, pertenecientes a 30 familias y 16 órdenes. El bosque xerofítico y la pastura exótica poseen un $52.7 \%$ de similitud en cuanto a la composición de las especies. Las especies registradas con mayor frecuencia 
fueron: Leptotila verreauxi (16.14\%), Ortalis canicollis (14.28 \%) y Furnarius cristatus (11.39\%). Las cámaras trampa resultaron efectivas para el registro del $33 \%$ de las especies identificadas a través de listas Mackinnon, y adicionaron dos especies. Por ello, resultan apropiadas para complementar métodos basados en la observación directa, particularmente para el registro de especies cinegéticas del ecosistema chaqueño pertenecientes a los órdenes Tinamiformes y Columbiformes.

Palabras clave: aves terrestres; fototrampeo; riqueza de especies; diversidad; Tinamiformes.

\section{REFERENCIAS}

Ahumada, J. A., Hurtado, J., \& Lizcano, D. (2013). Monitoring the status and trends of tropical forest terrestrial vertebrate communities from camera trap data: a tool for conservation. PloS one, 8(9), e73707. DOI: 10.1371/journal.pone.0073707

Angulo, F., Novoa, J., \& Lazo, I. (2016). Aves del río La Novia en la cuenca del Purús, Ucayali, Perú. En J. L. Mena \& C. Germaná (Eds.), Diversidad Biológica del Sudeste de la Amazonía Peruana: avances en la investigación (pp. 172-193). Perú, Lima: Consorcio Purús-Manu.

Armenteros, J. A., Prieto, R., Lomillos, J. M., Alonso, M. E., \& Gaudioso, V. R. (2015). Do wild Red-legged Partridges (Alectoris rufa) use feeders? An investigation of their feeding patterns using camera trapping. Avian Biology Research, 8(1), 14-24.

Arnulphi, V. C., Ortiz, S. G., \& Borghi, C. E. (2013). Características poblacionales del Cóndor Andino (Vultur gryphus) en el parque natural provincial Ischigualasto, Argentina. Ornitología Neotroprical, $24,1-5$.

Bayne, E., \& Hobson, K. (1997). Comparing the effects of landscape fragmentation by forestry and agriculture on predation of artificial nests. Conservation Biology, 11(6), 1418-1429.

Belda, A., Arques, J., Martínez, J., Peiró, V., \& Seva, E. (2009). Análisis de la biodiversidad de fauna vertebrada en el Parque Natural de la Sierra de Mariona mediante fototrampeo. Mediterranea: Serie de Estudios Biológicos, 20, 42-67.

Bradley, J., \& Marzluff, J. M. (2003). Rodents as nest predators: influences on predatory behavior and consequences to nesting birds. The Auk, 120(4), 1180-1187.

Buler, J., \& Hamilton, R. (2000). Predation of natural and artificial nests in a southern pine forest. The Auk, $117(3), 739-747$.

Caballero, J., Palacios, F., Arévalos, F., Rodas, O., \& Yanosky, A. (2014). Cambio de uso de la tierra en el
Gran Chaco Americano en el año 2013. Paraquaria Natural, 2(1), 21-28.

Clements, J. F., Schulenberg, T. S., Iliff, M. J., Roberson, D., Fredericks, T. A., Sullivan, B. L., \& Wood, C. L. (2016). The eBird/Clements checklist of birds of the world: v2016. Recuperado de http://www.birds. cornell.edu/clementschecklist/download/IOC

Colwell, R. K. (2013). EstimateS: Statistical estimation of species richness and shared species from samples (Versión 9). Recuperado de < purl.oclc.org/estimates>

Colyn, R. B., Campbell, A. M., \& Smit-Robinson, H. A. (2017). The application of camera trapping to assess Rallidae species richness within palustrine wetland habitat in South Africa. Ostrich, 88(3), 1-11.

Contreras, J., Agnolin, F., Davies, Y., Godoy, I., Giacchino, A., \& Ríos, E. (2014). Atlas Ornitogeográfico de la Provincia de Formosa, República Argentina ( $\left.{ }^{1 \mathrm{ra}} \mathrm{ed}.\right)$. Ciudad Autónoma de Buenos Aires, Argentina: Fundación de Historia Natural Félix de Azara.

Coria, R. D., Coria, O. R., \& Kunst., C. R. (2016). Diversidad y composición de especies de aves en un gradiente bosque-arbustal-sabana del chaco semiárido, Argentina. Ornitología Neotropical, 27, 1-15.

De La Peña, M. R., \& Krüger, E. (1987). Características ecológicas y algunos ambientes que frecuentan las aves argentinas. Santa Fe, Argentina: Facultad de Agronomía y Veterinaria.

Dinata, Y., Nugroho, A., Haidir, I. A., \& Linkie, M. (2008). Camera trapping rare and threatened avifauna in west-central Sumatra. Bird Conservation International, 18, 30-37.

Dinerstein, E., Olson, D., Graham, D., Webster, A., Bookbinder, M., \& Ledec, G. (1995). Una evaluación del estado de conservación de las ecorregiones terrestres de América Latina y el Caribe. Washington, D.C., USA: Banco Mundial.

Gascon, C., Lovejoy, T. E., Bierregaard Jr., R. O., Malcolm, J. R., Stouffer, P. C., Vasconcelos, H. L., ... Borges, S. (1999). Matrix habitat and species richness in tropical forest remnants. Biological Conservation, 91(2-3), 223-229.

González-García, F. (2012). Métodos para contar aves terrestres. En S. Gallina-Tesaro \& C. López-González (Eds.), Manual de técnicas para el estudio de la fauna (pp. 86-123). Querétaro, México: Universidad Autónoma de Querétaro.

Guerrero-Cárdenas, I., Galina-Tessaro, P., Álvarez-Cárdenas, S., \& Mesa-Zavala, E. (2012). Avistamientos recientes de águila real (Aquila chrysaetos) en la sierra El Mechudo, Baja California Sur, México. Revista Mexicana de Biodiversidad, 83, 397-401. 
Guyra Paraguay. (2005). Atlas de las Aves del Paraguay. Asunción, Paraguay: Guyra Paraguay.

Herrera, J. M. (2011). El papel de la matriz en el mantenimiento de la biodiversidad en hábitats fragmentados. De la teoría ecológica al desarrollo de estrategias de conservación. Ecosistemas, 20(2), 21-34.

IUCN. (2017, 27 de diciembre). The IUCN Red List of Threatened Species (Version 2017-3). Recuperado de $<$ http://www.iucnredlist.org $>$

Karanth, K., \& Nichols, J. (1998). Estimation of tiger densities in India using photographic captures and recaptures. Ecology, 79, 2852-2862.

Karanth, K., Nichols, J. D., Kumar, N. S., Link, W. A., \& Hines, J. E. (2004). Tigers and their prey: predicting carnivore densities from prey abundance. Proceedings of the National Academy of Sciences, 101(14), 4854-4858.

Li, S., Mcshea, W. J., Wang, D., Shao, L., \& Shi, X. (2010). The use of infrared-triggered cameras for surveying phasianids in Sichuan Province, China. Ibis, 152(2), 299-309.

Mastrangelo, M. E., \& Gavin, M. C. (2012). Trade-offs between cattle production and bird conservation in an agricultural frontier of the Gran Chaco of Argentina. Conservation Biology, 26(6), 1040-1051.

Mackinnon, J. R., \& Phillipps, K. (1993). A field guide to the birds of Borneo, Sumatra, Java, and Bali, the Greater Sunda Islands. United Kingdom, Oxford: Oxford University Press.

Mccallum, J. (2013). Changing use of camera traps in mammalian field research: Habitats, taxa and study types. Mammal Review, 43(3), 196-206.

Mena, J. L., Zúñiga Hartley, A., Villacorta Pezo, M., \& Salazar Zorrilla, S. (2016). Estimación de riqueza de mamíferos y aves terrestres de la cuenca alta del río La Novia, Purús a través de modelos de ocupación. En J. L. Mena \& C. Germaná (Eds.), Diversidad Biológica del Sudeste de la Amazonía Peruana: avances en la investigación (pp. 172-193). Perú, Lima: Consorcio Purús- Manu.

Mesa-Zavala, E., Alvarez-Cardenas, S., Galina-Tessaro, P., Troyo-Dieguez, E., \& Guerrero-Cardenas, I. (2012). Terrestrial vertebrates recorded by camera traps in areas with seasonal streams and creeks of superficial waters in a semiarid habitat of Baja California Sur, Mexico. Revista Mexicana de Biodiversidad, 83(1), 235-245.

Miura, S., Yasuda, M., \& Ratnam, L. (1997). Who steals the fruits? Monitoring frugivory of mammals in a tropical rain-forest. The Malayan Nature Journal, 50, 183-193.
Moreira, J., Mcnab, R. B., Thornton, D., García, R., PonceSantizo, G., \& Radachowsky, J. (2007). Abundancia de jaguares en La Gloria-El Lechugal, zona de usos múltiples, Reserva de la Biosfera Maya, Petén, Guatemala (Informe interno). Guatemala: Sociedad para la Conservación de la Vida Silvestre (WCS-Guatemala), Programa para la Conservación del Jaguar.

Moreno, C. E. 2001. Métodos para medir la biodiversidad. M \& T-Manuales y Tesis, (vol. 1). España, Zaragoza: Sociedad Entomológica Aragonesa (SEA).

Mosa, S. G., \& Goytia, M. (2004). Evaluación de la caza recreativa sobre la fauna silvestre en las provincias de Salta y Jujuy, Argentina. Memorias: Congreso Internacional sobre Manejo de Fauna Silvestre en la Amazonía y Latinoamérica, Iquitos, Perú. Recuperado de http://www.comfauna.org/wpcontent/uploads/2012/PDFs-Manejofaunasilvestre/ Iquitos-2004/6_Conservacion-uso-y-manejo-de-fauna-silvestre-por-comunidades/573-587_smosa_EvaluacionCazaRecreativaFaunaSilvestreSaltaJujuy.pdf

Mosquera-Muñoz, D., Corredor, G., Cardona, P., \& Armbrecht, I. (2015). Fototrampeo de aves caminadoras y mamíferos asociados en el piedemonte de Farallones de Cali. Boletín Cientifico Centro de Museos de Historia Natural, 18(2), 144-156.

Narosky, T., \& Yzurieta, D. (2006). Guía para la identificación de las Aves de Paraguay. Buenos Aires, Argentina: Vázquez Mazzini Editores.

O'Brien, T. G., \& Kinnaird, M. F. (2008). A picture is worth a thousand words: the application of camera trapping to the study of birds. Bird Conservation International, 18(S1), S144-S162.

O’Connell, A. F., Nichols, J. D., \& Karanth, K. U. (2010). Camera traps in animal ecology: methods and analysis. New York, USA: Springer

Pietz, P., \& Granfors, D. (2005). Parental nest defense on videotape: More reality than "myth". The Auk, 122(2), 701-705.

Ranganathan, J., Daniels, R. J. R., Chandran, M. D. S., Ehrlich, P. R., \& Daily, G. C. (2008). Sustaining biodiversity in ancient tropical countryside. Proceedings of the National Academy of Sciences, 105(46), 17852-17854.

Rojas-Robles, R., Stiles, G., \& Muñoz-Saba, Y. (2012). Frugivoría y dispersión de semillas de la palma Oenocarpus bataua (Arecaceae) en un bosque de los Andes colombianos. Revista de Biología Tropical, 60(4), 1445-1461.

Short, L. L. (1975). A zoogeographic analysis of the South American chaco avifauna. Bulletin of the American Museum of Natural History New York, 154(3), 1-352.

Tews, J., Brose, U., Grimm, V., Tielbörger, K., Wichmann, M. C., Schwager, M., \& Jeltsch, F. (2004). 
Animal species diversity driven by habitat heterogeneity/diversity: the importance of keystone structures. Journal of Biogeography, 31(1), 79-92.

Villareal, H., Álvarez, M., Córdoba, S., Escobar, F., Fagua G., Gast, F., \& Umaña, A. M. (2006). Manual de métodos para el desarrollo de inventarios de biodiversidad. Bogotá, Colombia: Instituto de Investigación de Recursos Biológicos Alexander von Humboldt.

Weiler, A., Esquivel, A., Peris, S., Silla, F., \& Salinas, P. (2018). Caracterización de la diversidad de aves en relación con los elementos del paisaje ganadero del Chaco Seco paraguayo. En A. Molin, E. Giustina, H.
Schmitz, L. Ribero, M. Pie, \& P. Löwenberg (Eds.), XXXII Congresso Brasileiro de Zoologia. Foz do Iguaçu, Brasil: Sociedade Brasileira de Zoologia.

Wemmer, C., Kunz, T. H., Lundie-Jenkins, G., \& McShea, W. J. (1996). Mammalian sign. En D. Wilson, F. Cole, J. Nichols, R. Rudran, \& M. Foster (Eds.), Meansuring and Monitoring Biological Diversity: Standard Methods for Mammals (pp. 157-176). Washington, USA: Smithsonian Institution Press.

Zetra, B., Rafiastanto, W., Rombang, M., \& Trainor, C. (2002). Rediscovery of the critically endangered Sumatran Ground Cuckoo Carpococcyx viridis. Forktail, 18, 63-65.

\section{APÉNDICE 1}

Especies de aves registradas mediante fototrampeo en la Estancia Montania, Boquerón, Paraguay detallando estado de residencia y su presencia en bosques xerofíticos y pasturas exóticas

\begin{tabular}{|c|c|c|c|c|c|}
\hline $\mathrm{N}^{\circ}$ & ORDEN/especies & Familia & Res & Bos & Pas \\
\hline \multicolumn{6}{|c|}{ TINAMIFORMES } \\
\hline 1 & Nothura chacoensis Conover, $1937^{(*)}$ & Tinamidae & $\mathrm{R}$ & & - \\
\hline 2 & Nothura boraquira (Spix, 1825) & Tinamidae & $\mathrm{R}$ & • & • \\
\hline 3 & Nothoprocta cinerascens (Burmeister, 1860) & Tinamidae & $\mathrm{R}$ & • & - \\
\hline 4 & Eudromia formosa (Lillo, 1905) & Tinamidae & $\mathrm{R}$ & & - \\
\hline 5 & Crypturellus tataupa (Temminck, 1815) & Tinamidae & $\mathrm{R}$ & • & • \\
\hline \multicolumn{6}{|c|}{ PELECANIFORMES } \\
\hline 6 & Butorides striata (Linnaeus, 1758) & Ardeidae & $\mathrm{R}$ & & • \\
\hline 7 & Ardea alba Linnaeus, 1758 & Ardeidae & $\mathrm{R}$ & • & • \\
\hline 8 & Ardea cocoi Linnaeus, 1766 & Ardeidae & $\mathrm{R}$ & • & • \\
\hline 9 & Nycticorax nycticorax (Linnaeus, 1758) & Ardeidae & $\mathrm{R}$ & & • \\
\hline 10 & Tigrisoma lineatum (Boddaert, 1783) & Ardeidae & $\mathrm{R}$ & • & $\bullet$ \\
\hline 11 & Egretta thula (Molina, 1782) & Ardeidae & $\mathrm{R}$ & • & \\
\hline 12 & Syrigma sibilatrix (Temminck, 1824) & Ardeidae & $\mathrm{R}$ & • & \\
\hline 13 & Theristicus caerulescens (Vieillot, 1817) & Threskiornithidae & $\mathrm{R}$ & • & • \\
\hline 14 & Theristicus caudatus (Boddaert, 1783) & Threskiornithidae & $\mathrm{R}$ & • & \\
\hline \multicolumn{6}{|c|}{ ANSERIFORMES } \\
\hline 15 & Chauna torquata (Oken, 1816) & Anhimidae & $\mathrm{R}$ & & • \\
\hline 16 & Callonetta leucophrys (Vieillot, 1816) & Anatidae & $\mathrm{R}$ & • & • \\
\hline 17 & Cairina moschata (Linnaeus, 1758) & Anatidae & $\mathrm{R}$ & & • \\
\hline \multicolumn{6}{|c|}{ CATHARTIFORMES } \\
\hline 18 & Coragyps atratus (Bechstein, 1793) & Cathartidae & $\mathrm{R}$ & • & • \\
\hline 19 & Cathartes aura (Linnaeus, 1758) & Cathartidae & $\mathrm{R}$ & - & \\
\hline \multicolumn{6}{|c|}{ ACCIPITRIFORMES } \\
\hline 20 & Buteogallus meridionalis (Latham, 1790) & Accipitridae & $\mathrm{R}$ & & • \\
\hline 21 & Buteogallus urubitinga (Gmelin, 1788) & Accipitridae & $\mathrm{R}$ & • & • \\
\hline 22 & Rupornis magnirostris (Gmelin, 1788) & Accipitridae & $\mathrm{R}$ & & • \\
\hline 23 & Geranospiza caerulescens (Vieillot, 1817) & Accipitridae & $\mathrm{R}$ & • & • \\
\hline
\end{tabular}


APÉNDICE 1 (Continuación) / APPENDIX 1 (Continued)

\begin{tabular}{|c|c|c|c|c|c|}
\hline $\mathrm{N}^{\circ}$ & ORDEN/especies & Familia & Res & Bos & Pas \\
\hline \multicolumn{6}{|c|}{ FALCONIFORMES } \\
\hline 24 & Caracara plancus (Miller, 1777) & Falconidae & $\mathrm{R}$ & • & • \\
\hline \multicolumn{6}{|c|}{ GALLIFORMES } \\
\hline 25 & Ortalis canicollis (Wagler, 1830) & Cracidae & $\mathrm{R}$ & $\bullet$ & • \\
\hline \multicolumn{6}{|c|}{ CARIAMIFORMES } \\
\hline 26 & Chunga burmeisteri (Hartlaub, 1860) & Cariamidae & $\mathrm{R}$ & • & - \\
\hline \multicolumn{6}{|c|}{ GRUIFORMES } \\
\hline 27 & Aramides ypecaha (Vieillot, 1819) & Rallidae & $\mathrm{R}$ & • & • \\
\hline 28 & Aramides cajanea (Müller, 1776) & Rallidae & $\mathrm{R}$ & • & \\
\hline 29 & Aramus guarauna (Linnaeus, 1766) & Aramidae & $\mathrm{R}$ & $\bullet$ & • \\
\hline \multicolumn{6}{|c|}{ CHARADRIIFORMES } \\
\hline 30 & Jacana jacana (Linnaeus, 1766) & Jacanidae & $\mathrm{R}$ & • & \\
\hline 31 & Vanellus chilensis (Molina, 1782) & Charadriidae & $\mathrm{R}$ & $\bullet$ & \\
\hline 32 & Tringa solitaria Wilson, 1813 & Scolopacidae & $\mathrm{N}$ & $\bullet$ & \\
\hline \multicolumn{6}{|c|}{ COLUMBIFORMES } \\
\hline 33 & Leptotila verreauxi Bonaparte, 1855 & Columbidae & $\mathrm{R}$ & $\bullet$ & • \\
\hline 34 & Columbina picui (Temminck, 1813) & Columbidae & $\mathrm{R}$ & $\bullet$ & • \\
\hline 35 & Columbina talpacoti (Temminck, 1810) & Columbidae & $\mathrm{R}$ & $\bullet$ & • \\
\hline 36 & Patagioenas picazuro (Temminck, 1813) & Columbidae & AS & $\bullet$ & • \\
\hline 37 & Patagioenas cayennensis (Bonnaterre, 1792) & Columbidae & $\mathrm{R}$ & • & \\
\hline 38 & Zenaida auriculata (Des Murs, 1847) & Columbidae & $\mathrm{R}$ & $\bullet$ & • \\
\hline \multicolumn{6}{|c|}{ PSITTACIFORMES } \\
\hline 39 & Myiopsitta monachus (Boddaert, 1783) & Psittacidae & $\mathrm{R}$ & & • \\
\hline \multicolumn{6}{|c|}{ CUCULIFORMES } \\
\hline 40 & Guira guira (Gmelin, 1788) & Cuculidae & $\mathrm{R}$ & • & • \\
\hline 41 & Crotophaga ani Linnaeus, 1758 & Cuculidae & $\mathrm{R}$ & & • \\
\hline 42 & Crotophaga major Gmelin, 1788 & Cuculidae & $\mathrm{AN}$ & & • \\
\hline \multicolumn{6}{|c|}{ STRIGIFORMES } \\
\hline 43 & Bubo virginianus (Gmelin, 1788) & Strigidae & $\mathrm{R}$ & • & \\
\hline \multicolumn{6}{|c|}{ PICIFORMES } \\
\hline 44 & Colaptes melanochloros (Gmelin, 1788) & Picidae & $\mathrm{R}$ & $\bullet$ & • \\
\hline \multicolumn{6}{|c|}{ PASSERIFORMES } \\
\hline 45 & Drymornis bridgesii (Eyton, 1849) & Dendrocolaptidae & $\mathrm{R}$ & & • \\
\hline 46 & Xiphocolaptes major (Vieillot, 1818) & Dendrocolaptidae & $\mathrm{R}$ & • & • \\
\hline 47 & Lepidocolaptes angustirostris (Vieillot, 1818) & Dendrocolaptidae & $\mathrm{R}$ & & • \\
\hline 48 & Phacellodomus sibilatrix Sclater, 1879 & Furnariidae & $\mathrm{R}$ & & • \\
\hline 49 & Furnarius cristatus Burmeister, 1888 & Furnariidae & $\mathrm{R}$ & • & • \\
\hline 50 & Furnarius rufus (Gmelin, 1788) & Furnariidae & $\mathrm{R}$ & • & • \\
\hline 51 & Asthenes pyrrholeuca (Vieillot, 1817) & Furnariidae & AV & $\bullet$ & \\
\hline 52 & Coryphistera alaudina Burmeister, 1860 & Furnariidae & $\mathrm{R}$ & • & - \\
\hline 53 & Taraba major (Vieillot, 1816) & Thamnophilidae & $\mathrm{R}$ & • & • \\
\hline 54 & Thamnophilus doliatus (Linnaeus, 1764) & Thamnophilidae & $\mathrm{R}$ & & • \\
\hline 55 & Pitangus sulphuratus (Linnaeus, 1766) & Tyrannidae & $\mathrm{R}$ & • & • \\
\hline 56 & Elaenia spectabilis Pelzeln, 1868 & Tyrannidae & $\mathrm{AN}$ & & • \\
\hline 57 & Myiodynastes maculatus (Müller, 1776) & Tyrannidae & $\mathrm{AN}$ & & - \\
\hline 58 & Fluvicola albiventer (Spix, 1825) & Tyrannidae & $\mathrm{R}$ & • & • \\
\hline 59 & Pyrocephalus rubinus (Boddaert, 1783) & Tyrannidae & AS & • & \\
\hline
\end{tabular}


APÉNDICE 1 (Continuación) / APPENDIX 1 (Continued)

\begin{tabular}{|c|c|c|c|c|c|}
\hline $\mathrm{N}^{\circ}$ & ORDEN/especies & Familia & Res & Bos & Pas \\
\hline 60 & Machetornis rixosa (Vieillot, 1819) & Tyrannidae & $\mathrm{R}$ & • & \\
\hline 61 & $\begin{array}{l}\text { Knipolegus striaticeps (D’Orbigny \& Lafresnaye, } \\
\text { 1837) }\end{array}$ & Tyrannidae & AS & & $\cdot$ \\
\hline 62 & Cyanocorax chrysops (Vieillot, 1818) & Corvidae & $\mathrm{R}$ & & • \\
\hline 63 & Mimus triurus (Vieillot, 1818) & Mimidae & AV & • & • \\
\hline 64 & Turdus amaurochalinus Cabanis, 1851 & Turdidae & $\mathrm{R}$ & • & • \\
\hline 65 & Cyclarhis gujanensis (Gmelin, 1789) & Vireonidae & $\mathrm{R}$ & • & \\
\hline 66 & Saltator coerulescens Vieillot, 1817 & Thraupidae & $\mathrm{R}$ & • & • \\
\hline 67 & Saltator aurantiirostris Vieillot, 1817 & Thraupidae & $\mathrm{R}$ & • & • \\
\hline 68 & Saltatricula multicolor (Burmeister, 1860) & Thraupidae & $\mathrm{R}$ & & • \\
\hline 69 & Paroaria coronata (Miller, 1776) & Thraupidae & $\mathrm{R}$ & • & • \\
\hline 70 & Coryphospingus cucullatus (Müller, 1776) & Thraupidae & $\mathrm{R}$ & • & • \\
\hline 71 & Sicalis flaveola (Linnaeus, 1766) & Thraupidae & $\mathrm{R}$ & • & \\
\hline 72 & Cyanoloxia brissonii (Lichtenstein, 1823) & Cardinalidae & $\mathrm{R}$ & • & • \\
\hline 73 & Agelaioides badius (Vieillot, 1819) & Icteridae & $\mathrm{R}$ & • & • \\
\hline 74 & Cacicus solitarius Vieillot, 1816 & Icteridae & $\mathrm{R}$ & $\bullet$ & $\cdot$ \\
\hline
\end{tabular}

(*) Considerada subespecie de Nothura maculosa por algunos autores.

Res = Estado de residencia; Bos = bosque xerofítico; Pas $=$ pastura exótica; $\mathrm{R}=$ Residente nidificante permanente (reproductor residente de todo el año en Paraguay); AV = Visitante Invernal (no nidifica en Paraguay, arriban desde el sur u oeste en invierno); AS = Nidificante Migrante del Sur (nidifica en Paraguay, más abundantes en invierno); $\mathrm{AN}=\mathrm{Migrador}$ Austral (nidifica en Paraguay durante el verano austral y migra a latitudes tropicales durante el invierno).

Res $=$ State of residence; Bos $=$ xerophytic forest Pas = exotic pasture; $\mathrm{R}=$ permanent nesting resident (all year long nesting resident in Paraguay); AV = Winter Visitor (does not nest in Paraguay, arrives from south or west in winter); AS = Southern Migrant Nesting (nest in Paraguay, more abundant in winter); AN = Austral Migrator (nest in Paraguay during southern summer and migrates to tropical latitudes during winter). 